\title{
GROUPS OF AUTOMORPHISMS OF ALMOST KAEHLER MANIFOLDS
}

\author{
BY S. I. GOLDBERG ${ }^{1}$ \\ Communicated by S. Bochner, January 9,1960
}

1. Let $M$ be a compact almost Kaehler manifold ${ }^{2}$ of real dimension $2 n$. The fundamental 2 -form $\omega$ (which together with the metric $g$ of $M$ defines the almost Kaehlerian structure) is harmonic and therefore invariant by every infinitesimal isometry [2]. Let $X$ be an infinitesimal conformal transformation of $M$. Then, for all $n>1$ we shall show that $X$ is in fact an infinitesimal isometry. Indeed, the following theorem is proved:

THEOREM 1. The largest connected Lie group of conformal transformations of a compact almost Kaehler manifold $M^{2 n}(n>1)$ coincides with the largest connected group of automorphisms of the almost Kaehlerian structure. Moreover, the infinitesimal automorphisms are infinitesimal isometries.

This generalizes a previous result [3]. If the almost complex structure is completely integrable and comes from a complex analytic structure we obtain the theorem of Lichnerowicz [5] whose methods it seems cannot be extended to include the almost Kaehler manifolds.

In the noncompact case if we consider infinitesimal conformal maps whose covariant forms are closed, a much wider class of manifolds may be considered.

2. Let $X$ be a vector field on $M$ whose image by the almost complex structure operator $J$ is "closed," that is, its covariant form $C \xi$ is closed where $C$ is the complex structure operator applied to forms. Then, $X$ is an infinitesimal automorphism of $M$. Denote by $t(X)$ the tensorfield $\theta(X) J$ modulo $i(X) D \omega$ where $\theta(X), i(X)$ and $D$ are the Lie derivative, interior product and covariant differential operators, resp. For Kaehler manifolds $D \omega$ vanishes, and so $t(X)$ and $\theta(X) J$ coincide. In this case, the vanishing of $t(X)$ characterizes the infinitesimal analytic transformations. Let $t$ be a covariant real tensor of order 2 and denote by $J$ again the operator

1 This research was supported by the United States Air Force Office of Scientific Research of the Air Research and Development Command under Contract No. AF49(638)-14.

${ }^{2}$ The manifolds, differential forms and tensor fields considered are assumed to be of class $C^{\infty}$. 


$$
J: t_{i j} \rightarrow t_{i r} w_{j}^{r}, \quad \omega=w_{i j} d x^{i} \wedge d x^{j} .
$$

Clearly, $J \omega=g$. For any vector field $X$ on a Kaehler manifold it can be shown that

$$
\bar{\theta}(X) \omega-\theta(X) \omega=\delta \xi \cdot \omega-2 \tilde{t}(X)
$$

where $\bar{\theta}(X)$ denotes the dual of $\theta(X)$ and $\tilde{t}(X)$ denotes the 2 -form corresponding to the skew-symmetric part of $t(X)$ [3].

Lemma 1. For any vector field $X$ on a Kaehler manifold $M$

$$
\|\theta(X) \omega\|^{2}=\|\delta \xi\|^{2}+2(\tilde{t}(X), \theta(X) \omega)
$$

where $\|\alpha\|^{2}=(\alpha, \alpha)=\int_{M} \alpha \wedge^{*} \alpha$ for any $p$-form $\alpha$.

Theorem 2. A vector field $X$ defines an infinitesimal analytic transformation of a Kaehler manifold if and only if $J \theta(X) \omega=\theta(X) g$, that is when applied to $\omega$ the operators $\theta(X)$ and $J$ commute.

This follows from the fact that $t(X)=\theta(X) \omega+J \theta(X) g$ a relation used in establishing formula (1). Lemma 1 therefore implies the following

Corollary. For an infinitesimal analytic transformation

$$
\|\theta(X) \omega\|=\|\delta \xi\| \text {. }
$$

Hence, a divergence free analytic map is an infinitesimal automorphism of the Kaehler structure.

Lemma 2. For an infinitesimal conformal transformation $X$ of a Kaehler manifold

$$
t(X)=\theta(X) \omega+\frac{1}{n} \delta \xi \cdot \omega .
$$

Our notation does not distinguish between the 2-form $t(X)$ and the corresponding tensorfield. If $\xi$ is closed, $t(X)$ is symmetric and must therefore vanish. We conclude from the lemma that $d \delta \xi=0$ for $n>1$, that is $X$ is homothetic. Since a homothetic map of a complete Riemannian manifold which is not locally flat is isometric we conclude

THEOREM 3. A closed infinitesimal conformal transformation of a complete Kaehler manifold $M^{2 n}(n>1)$ which is not locally flat is an automorphism of the Kaehler structure.

In the locally flat case an infinitesimal affine transformation $X$ is isometric if and only if its length is bounded, that is the vector field on $M$ defining $X$ has bounded length. Hence, since a homothetic map is affine we have 
Theorem 4. A closed infinitesimal conformal map of a complete locally flat Kaehler manifold $M^{2 n}(n>1)$ is an automorphism of the Kaehler structure if and only if its length is bounded.

Remarks. (a) Every conformal map of a complete flat space is homothetic.

(b) M. Obata has communicated to us the following result (unpublished): "A closed infinitesimal conformal transformation of a (locally) reducible Riemannian manifold is homothetic." This means that only an irreducible Riemannian manifold can admit closed nonhomothetic maps.

3. Proof of Theorem 1. It is first shown that

$$
\theta(X) \omega+\bar{\theta}(X) \omega=\left(1-\frac{2}{n}\right) \delta \xi \cdot \omega .
$$

Since $\bar{\theta}(X)=\epsilon(\xi) \delta+\delta \epsilon(\xi)$ (where $\epsilon(\xi) \alpha=\xi \wedge \alpha$ for any $p$-form $\alpha$ ), $\delta$ and $\bar{\theta}(X)$ commute. Hence, applying $\delta$ to both sides of (3) we obtain

$$
\delta \theta(X) \omega=-\left(1-\frac{2}{n}\right) C d \delta \xi .
$$

Taking the global scalar product with $C \xi$ we derive

$$
\|\theta(X) \omega\|^{2}=-\left(1-\frac{2}{n}\right)\|\delta \xi\|^{2} .
$$

For $n>1, \theta(X) \omega$ vanishes. Moreover, $\delta \xi=0$, that is the automorphisms are isometries.

4. Bochner and Montgomery [1] have shown that the group $G$ of analytic homeomorphisms of a compact complex manifold $M$ is a Lie group. If $M$ is an Einstein Kaehler manifold $G$ is reductive [6]. More generally, if the Ricci scalar curvature of a compact Kaehler manifold is a (positive) constant the same conclusion is valid [5]. This seems to be the best possible generalization of the result of [6] as one may see by considering the Gaussian 2-sphere with any metric with nonconstant scalar curvature. By restricting the analytic maps to those which are closed in the above sense no restrictions of a local nature regarding curvature are required and results parallel to Theorems 3 and 4 may be obtained.

REMARK. From the proof of Theorem 3 it follows that the image by $J$ of a closed infinitesimal conformal transformation of a Kaehler manifold is an infinitesimal isometry. In fact 
Theorem 5. A closed infinitesimal conformal transformation of a Kaehler manifold is an infinitesimal analytic transformation whose image by $J$ is an infinitesimal isometry.

For noncompact almost Kaehler manifolds we may prove

THEOREM 6. If the largest connected group of automorphisms is a semi-simple Lie group its elements are volume preserving transformations.

\section{REFERENCES}

1. S. Bochner and D. Montgomery, Groups of differentiable and real or complex analytic transformations, Ann. of Math. vol. 46 (1945) pp. 685-694.

2. S. Bochner and K. Yano, Curvature and Betti numbers, Ann. Math. Studies vol. 32 (1953) p. 49.

3. S. I. Goldberg, Groups of automorphisms of Kaehler manifolds, AFOSR technical note 59-727.

4. S. Ishihara and M. Obata, Affine transformations in a Riemannian manifold, Tôhoku Math. J. vol. 7 (1955) pp. 146-150.

5. A. Lichnerowicz, Sur les transformations analytiques des variêtés kählériennes compactes, C. R. Acad. Sci. Paris vol. 244 (1957) pp. 3011-3014.

6. Y. Matsushima, Sur la structure du groupe d'homéomorphismes analytiques d'une certaine variété kählérienne, Nagoya Math. J. vol. 11 (1957) pp. 145-150.

Wayne State University 